\title{
KLF14 Inhibits Renal Mesangial Cell Proliferation by Promoting Btg2 Gene Expression
}

\section{Xiaoyuan Wu}

Department of Nephrology, Beijing Tiantan Hospital, Capital Medical University

\section{Yuxiang Ma}

Department of Nephrology, Beijing Chuiyangliu Hospital affiliated to Tsinghua University

\section{Xiaoying Lian}

Department of Nephrology, Beijing Luhe Hospital, Capital Medical University

\section{Shupeng Lin}

Department of Nephrology, Chinese PLA General Hospital, State Key Laboratory of Kidney Diseases, National Clinical Research Center for Kidney Diseases, Beijing Key Laboratory of Kidney Diseases

\section{Shunlai Shang}

Department of Nephrology, Chinese PLA General Hospital, State Key Laboratory of Kidney Diseases, National Clinical Research Center for Kidney Diseases, Beijing Key Laboratory of Kidney Diseases

Xueyuan Bai ( $\nabla$ xueyuan_bai@163.com )

Department of Nephrology, Chinese PLA General Hospital, State Key Laboratory of Kidney Diseases, National Clinical Research Center for Kidney Diseases, Beijing Key Laboratory of Kidney Diseases

\section{Research Article}

Keywords: Krüppel-like factor 14, mesangial cell, proliferation, B-cell translocation gene 2 (Btg2)

Posted Date: January 13th, 2021

DOI: https://doi.org/10.21203/rs.3.rs-122817/v1

License: @ (i) This work is licensed under a Creative Commons Attribution 4.0 International License. Read Full License 


\begin{abstract}
Krüppel-like factor 14 is one of zinc finger protein family that are closely associated with regulation of transcription, and its function in the kidneys remains unclear. So KLF14-deficient mouse models were established using TALEN. The levels of blood cholesterol, HDL-C, LDL-C, and TGs were significantly decreased in KLF14-/- mice. KLF14-/- mice had more obvious mesangial cell proliferation and greater accumulation of extracellular matrix. RNA-Seq showed that these differentially expressed genes were mainly involved in metabolism, proliferation and inflammation pathways. ChIP-Seq was performed to identify KLF14 target genes. Five overlapping KLF14 target genes (Btg2, Socs2, Hdc, Ler2 and Akr1b3) were identified by combined analyses of RNA-Seq and ChIP-SEq. Dual luciferase reporter assay and EMSA confirmed that Btg2 was a target gene of KLF14. EdU assay revealed that KLF14 could inhibit the proliferation of primary renal mesangial cells by promoting Btg2 expression. KLF14 might exert its function in kidneys by regulating metabolism, proliferation, and inflammation signaling pathways. KLF14 inhibited the proliferation of primary RMCs by promoting expression of its target gene Btg2. This study provides a basis for further functional studies of KLF14 in the development of kidney diseases, especially in mesangial proliferative glomerulonephritis, metabolic nephropathy and kidney tumors.
\end{abstract}

\title{
Introduction
}

The Krüppel-like factor (KLF) gene family is a group of zinc finger proteins that are closely associated with the transcription regulation in eukaryotes[1-2]. The carboxyl terminus of KLF proteins is highly conserved. It contains three tandem Cys2/His2 zinc finger structures for DNA binding. The amino terminus has larger differences among different family members and mainly participates in transcriptional regulation by binding to co-factors [3-6]. The expression of KLFs has tissue, cell distribution, and developmental stage specificity. KLFs regulate the expression of GC or CACCC-rich promoter genes to participate in many physiological and pathological processes, including cell proliferation, differentiation, and apoptosis as well as tumor development and progression [5-8]. Krüppel-like factor 14 (KLF14) is a newly discovered member of this family encoded by an intronless gene and displays monoallelic maternal expression in all studied tissues in both humans and mouse [9].

Recently, a large-scale genome-wide association study (GWAS) has shown that KLF14 variations are closely associated with type 2 diabetes mellitus and the level of high-density lipoprotein cholesterol (HDL-C) [10-12]. KLF14 knockout mice can exhibit centrosome amplification, chromosome aneuploidy, and spontaneous tumorigenesis [13]. However, it is currently unclear whether KLF14 plays an important role in the development of kidney diseases.

To determine the function of KLF14 in kidneys, in this study, we knocked out the KLF14 gene in mice using the gene editing technology transcription activator-like effector nuclease (TALEN) to establish a KLF14-deficient mouse models. The changes in blood biochemical indicators, kidney function and kidney pathology in KLF14-deficient mice were observed. Next, the differences in gene expression profiles in kidney tissues between knockout and wild-type mice were compared using RNA-sequencing (RNA-Seq). The KLF14 target genes were screened using chromatin immunoprecipitation-sequencing (ChIP-Seq), and the KLF14 target gene, B-cell translocation gene 2 (Btg2), was further validated using the dual-luciferase reporter assay and electrophoretic mobility shift assay (EMSA). Finally, the specific mechanisms by which KLF14 modulated the proliferation of glomerular mesangial cells were investigated in vitro using mouse primary renal mesangial cells (RMCs).

\section{Results}

\section{Establishment of the KLF14-deficient (KLF14-/-) mouse models and changes in blood and urine biochemical indicators}

To study the function of KLF4, we used the genome editing technology TALEN to establish a KLF14 knockout (KO) mouse models. DNA from tails of KLF14-/- mice and KLF14+/+ mice was extracted for gene sequencing identification. The sequencing results revealed that four base deletions in the genome of KLF14-/- compared with KLF14+/+ mice (Fig. S1 A-B). The expression level of KLF14 in the kidneys of KLF14-/- and KLF14+/+ mice was detected by using western blot. The results showed that compared with KLF14+/+ mice, the kidneys of KLF14-/- mice did not express KLF14 at all (Fig. S1 C).

To determine the role of KLF14 in mice, blood and 24-h urine samples from KLF14-/- and KLF14+/+ mice were collected to compare the differences between these two groups. The results showed that the levels of cholesterol, HDL-C, LDL-C, and TGs were significantly lower in the KLF14-/- group than the KLF14+/+ group $(\mathrm{p}<0.05)$. Urinary albumin/creatinine ratio, blood creatinine, blood albumin, and blood sugar levels did not show significant differences between KLF14-deficient (KLF14-/-) mice and wild-type (KLF14+/+) mice (Table 1). The

Page 2/13 
average level of the urine albumin excretion rate and insulin in the KLF14-/- group was higher than that in the wild-type group; however, there was no significant difference.

Table 1

Changes in blood and urine biochemical indicators in KLF14-/-mice

\begin{tabular}{|c|c|c|c|c|c|c|c|c|c|c|}
\hline Gp & $\begin{array}{l}\text { ALB } \\
(\mathrm{g} / \mathrm{L})\end{array}$ & $\begin{array}{l}\text { CHOL } \\
(\mathrm{mmol} / \mathrm{L})\end{array}$ & $\begin{array}{l}\text { CREA } \\
\text { (umol/L) }\end{array}$ & $\begin{array}{l}\text { GLU } \\
(\mathrm{mmol} / \mathrm{L})\end{array}$ & $\begin{array}{l}\text { HDL-c } \\
(\mathrm{mmol} / \mathrm{L})\end{array}$ & $\begin{array}{l}\text { LDL-c } \\
(\mathrm{mmol} / \mathrm{L})\end{array}$ & $\begin{array}{l}\text { TG } \\
(\mathrm{mmol} / \mathrm{L})\end{array}$ & $\begin{array}{l}\text { UREA } \\
\text { (mmol/L) }\end{array}$ & $\begin{array}{l}\text { Insulin } \\
\text { (ug/L) }\end{array}$ & $\begin{array}{l}\text { ACR } \\
(\mathrm{g} / \mathrm{mmol})\end{array}$ \\
\hline \multirow{2}{*}{$\begin{array}{l}\text { KLF14 } \\
+/+\end{array}$} & $30.70 \pm$ & $7.49 \pm$ & $20.73 \pm$ & $7.76 \pm$ & $2.73 \pm$ & $5.01 \pm$ & $1.06 \pm$ & $11.29 \pm$ & $0.62 \pm$ & $0.45 \pm$ \\
\hline & 1.33 & 1.44 & 11.76 & 1.50 & 0.29 & 1.43 & 0.29 & 0.64 & 0.35 & 0.06 \\
\hline \multirow[t]{2}{*}{ KLF14-/- } & 31.67 & $2.08 \pm$ & $20.0 \pm$ & $5.83 \pm$ & $1.8 \pm$ & $0.61 \pm$ & $0.46 \pm$ & $13.10 \pm$ & $0.94 \pm$ & $0.84 \pm$ \\
\hline & & $0.40^{*}$ & 14.94 & 3.65 & $0.45^{\star}$ & $0.280 *$ & $0.20 *$ & 7.85 & 0.79 & 0.22 \\
\hline
\end{tabular}

ALB: albumin, CHOL: cholesterol, CREA: creatinine, GLU: glucose, HDL-c: high-density lipoprotein cholesterol, LDL-c: low-density lipoprotein cholesterol, TG: triglyceride, UREA: urea, ACR: urinary albumin /creatinine ratio. All the values are expressed as Mean \pm SEM. * $\mathrm{p}<0.05$ vs KLF14 +/+group.

\section{Pathological changes in kidney tissues of KLF14-/- mice}

PAS staining was performed to assess the pathological morphological changes in kidney tissues of KLF14-/- mice. The results showed that the KLF14-/-group had a heavier accumulation of extracellular matrix and more marked renal mesangial cell proliferation per glomerulus compared with KLF14-/- mice. This difference was statistically significant (Fig. 1A-1B).

To clarify the inhibition effect of KLF14 for proliferation in kidneys, we used PCNA to detect glomerular proliferation in both groups by immunohistochemical staining. The numbers of PCNA-positive cells were counted in at least 20 glomeruli per mouse kidney section to quantify the degree of cell proliferation. The results showed that KLF14-/- mice expressed a higher percentage of PCNA-positive cells than $\mathrm{KLF} 14+/+$ mice $(\mathrm{p}<0.05)($ Fig. 1C-1D). These results suggested that KLF14 played a regulatory role in the proliferation process in kidneys.

\section{Changes in the gene expression profile in the kidney of KLF14 -/- mice}

To clarify the physiological function of the transcription factor KLF14 in mouse kidney, we performed RNA-Seq analysis for RNA isolated from kidney tissues of KLF14+/+ and KLF14-/- mice. Interestingly, we found 38 differentially expressed genes (DEGs) in the kidney samples when KLF14-/- mice were compared to KLF14+/+ mice. In KLF14-/- mice, the expression levels of 12 genes were significantly up-regulated, and the expression levels of 26 genes were significantly down-regulated (Table S1). These DEGs mainly participated in biological processes, including the regulation of proliferation, lipid metabolic process, immune system process and immune response, and response to corticosteroid (Fig. 2A). Among these DEGs, Btg2, Fos, Psca, Cdkn1a, and Dusp1 were associated with proliferation, Ccl28, Socs2, Jchain, and Cfd were associated with immune inflammation, and Akr1b3, Akr1c18, Hmgcr, and Hdc were associated with metabolism.

To validate the accuracy of the RNA-Seq analyses, 10 differentially expressed genes were randomly selected for qRT-PCR validation. qRTPCR and RNA-Seq showed similar results, with seven genes (Btg2, Akr1b3, Hdc, ler2, Socs2, Fosb, and Ccl28) showing down-regulated expression and three genes (Tmem254b, S100a9, and Cfd) showing up-regulated expression (Fig. 2B).

\section{GO and KEGG Pathway analyses of differentially expressed genes (DEGs) in the kidneys of KLF14-/- mice}

To obtain a broad assessment of the molecular functions, biological processes, and cellular components of these 38 DEGs, we performed GO enrichment analysis. The enriched pathways were analyzed using the KEGG pathway database. The GO analysis was classified into three functional groups: molecular function (MF), biological process (BP), and cellular component (CC). Enrichment analysis of the DEGs was performed using the GO term database (http://www.geneontology.org/). The GO analysis showed that among the 38 DEGs with known classes of biological process, 21 genes were identified to be involved in metabolic process and response to stimulus, and 10 genes in immune system process and signaling transduction. For MF, the majority of the up- and down-regulated DEGs were clustered in binding activity and catalytic activity. For CC, up- and down-regulated genes were mainly enriched intracellularly (Fig. S2). 
Based on the KEGG pathway enrichment analysis, we found that the pathways with significant enrichment of the DEGs in kidney tissues of KLF14-/- mice mainly were tumor and proliferation-related pathways, metabolism and endocrine-related pathways, immune inflammation-related pathways, and signal transduction pathways (Fig. S3). Tumor and proliferation pathways with significant enrichment included the mitogen-activated protein kinase (MAPK) signaling pathway, forkhead box 0 (FoxO) signaling pathway, Janus kinase-signal transducer and activator of transcription (Jak-STAT) signaling pathway, transcriptional misregulation in cancer, phosphatidylinositol 3-kinase-protein kinase B (PI3K-Akt) signaling pathway, and the cell cycle. The metabolism-related pathways with significant enrichment mainly included terpenoid backbone biosynthesis, histidine metabolism, and type II diabetes mellitus. The immune inflammation-related pathways with significant enrichment included complement and coagulation cascades, tumor necrosis factor (TNF) signaling pathway, and intestinal immune network for immunoglobulin A (IgA) production (Fig. S4).

\section{Results of screening of KLF14 target genes using ChIP-Seq}

To further search for the target genes of KLF14, the ChIP-Seq experiment was performed on the kidneys of wild-type mice. The specific binding DNA sequence of KLF14 was precipitated by ChIP. Since SK1 is known to be one of the target genes of KLF14, the promoter sequence of SK1 was amplified by ChIP products. The precipitated DNA sequence includes SK1 promoter region, which proves the reliability of ChIP products (Fig. S4). A total of 24042216 clean sequencing reads were obtained in the KLF14 experimental group and 22447160 clean sequencing reads in the input control group. Compared with the mouse genome (mm9), a total of 21750434 and 20815816 clean sequencing reads were obtained. These two groups of data were compared using MACS software, and the results showed that 2621 sequence peaks might be the binding regions of KLF14 in the genome.

GO and KEGG pathway analyses were performed on the ChIP-Seq results. The GO analysis results showed that biological processes with greater involvement of peak related genes were cellular process and metabolic process (Fig. S5). KEGG pathway analysis results showed that pathways with peak related gene involvement were mainly proliferation and tumor-related, metabolism-related, and immune inflammation-related pathways, of which metabolism pathways were mainly global and overview maps as well as lipid metabolism (Fig. S6). Table S2 shows the pathway categories with significant changes in the KEGG pathway. These pathways played important roles in the regulation of KLF14.

To further identify KLF14 target genes, the RNA-Seq and ChIP-Seq results were combined for analysis. As shown in Fig. 4A, there were five overlapping target genes between the RNA-Seq and ChIP-Seq: Socs2, Btg2, Akr1b3, Hdc, and ler2. Analyses using the JASPAR website (http://jaspar.genereg.net/) showed that the promoter regions of these five genes all contained KLF14 binding regions. To perform an in-depth analysis of Socs2, Btg2, Akr1b3, Hdc, and ler2, we conducted gene Pathway Network analysis for these five elements. The Pathway Network analysis showed that the five KLF14-regulated target genes were related to inflammation, type II diabetes mellitus, proliferation, lipid metabolic process, histidine metabolism, and signal transduction pathways, respectively (Fig. 4B).

\section{Confirmation of Btg2 as a target gene of transcription factor KLF14 using dual-luciferase reporter assay and EMSA}

To confirm the role of KLF14 in Btg2 promoter activity, the KLF14 eukaryotic expression vector (pCMV6-AC-GFP-KLF14) or KLF14 vector control (pCMV6-AC-GFP), Btg2 promoter luciferase reporter gene plasmid (pGL3-Basic-Btg2 promoter) or Btg2 control plasmid (pGL3Basic), and renilla luciferase reporter gene plasmid (pGMLR-TK luciferase reporter plasmid) were co-transfected into HEK293 cells. The results showed that KLF14 overexpression significantly increased the relative luciferase activity of pGL3-Basic-Btg2 promoter plasmids (Fig. 5A).

The KLF14 binding site was further confirmed by EMSA of nuclear extracts from primary MRMCs. As shown in Fig. 5B, the nuclear extracts were combined with the biotin-labeled probe (lane 3). The combined rates of the nuclear extract and KLF14 binding site were increased in the KLF14 overexpression group (lane 2). A competition assay showed that pre-incubation with a 100-fold molar excess of cold probe (lane 4), but not cold mutated probe, diminished the intensity of the bands (lane 5). The results demonstrated that the KLF14 transcription factor could combine directly with the binding sites in Btg2 promoter nucleotides.

\section{KLF14 Inhibited the proliferation of cultured mouse mesangial cells in vitro through activation of Btg2}


In vivo experiments had confirmed that KLF14 deficiency aggravated the proliferation of glomerular mesangial cells. To explore the direct role of KLF14 in mesangial cell proliferation and the underlying mechanisms, the mouse KLF14 eukaryotic expression vector pCMV6-ACGFP-KLF14 and Btg2 short interfering RNA (siRNA) were transfected into cultured primary mouse renal mesangial cells (MRMCs) in vitro. MRMCs were starved in serum-free medium for $24 \mathrm{~h}$ to deplete growth factors and then transfected with pCMV6-AC-GFP-KLF14 + Btg2 siRNA (KLF14 + Btg2 siRNA group), pCMV6-AC-GFP-KLF14 plasmid + unrelated siRNA control (KLF14 group), or KLF14 control plasmid pCMV6-AC-GFP (control group). The proliferative level of MRMCs was observed $48 \mathrm{~h}$ after transfection. As shown in Fig. 6, the percentage of EdU-positive cells in the KLF14 group was significantly decreased compared with the control group, and the EdU positivity rate in the KLF14 + Btg2 siRNA group significantly increased compared with the KLF14 group, which was similar to that in the KLF14 control plasmid group (control group). These results indicated that KLF14 functioned to inhibit mesangial cell proliferation through the activation of Btg2.

Btg2 is an immediate early response gene. When cells are stimulated by genotoxic drugs, oxidative stress, and ionizing radiation, Btg2 expression is up-regulated through p53-dependent or independent pathways to act on a series of downstream target molecules, causing cells to arrest at G1/S phase, which is the major functional pathway of the Btg2 gene. Therefore, we conducted the Western Blot experiment to check the changes in the expression of cell cycle regulatory proteins and Btg2 in the renal tissue of KLF14-/- mice. The results showed that the expression level of Btg2 in KLF14-/-mice significantly decreased, the expression level of Cyclin B1, Cyclin D1, CDK2 increased significantly in KLF14-/-mice. This indicates that Btg2 might exert its function through influencing the expression of Cyclin B1, Cyclin D1, and CDK2.

\section{Discussion}

To study the function of KLF14, we used the genome editing technology TALEN to establish a KLF14 gene knockout mouse models. The results showed that the levels of blood cholesterol, HDL-C, LDL-C, and TG in KLF14-/- mice significantly decreased compared with the $\mathrm{KLF} 14+/+$ group. The pathological results showed that the KLF14 knockout could aggravate glomerular proliferation. Similar to the histological manifestations, the number of glomerular nucleated cells increased more noticeably in the KLF14-/- mice.

To investigate the role of KLF14 in mouse kidneys, the differences in mRNA expression profiles between the kidney tissues of KLF14+/+ and KLF14-/- mice were analyzed using RNA-SEq. Compared with wild-type mice, we observed significant changes in mRNA expression in the kidneys of KLF14-/- mice. Among these differentially expressed mRNAs, most mRNAs were down-regulated, and a few mRNAs were up-regulated. Biological processes that involved these differentially expressed genes (DEGs) focused mainly on the regulation of proliferation, lipid metabolic process, immune system process and immune response, and response to corticosteroid.

To gain further insights into the role of KLF14 in kidneys, the target genes of KLF14 in kidneys were determined using ChIP-SEq. The biological function and pathways of these targets were analyzed. The results revealed that the peak related genes in kidneys mainly regulated the pathways or genes associated with metabolism, cell proliferation, immune pathways, and extracellular matrix (ECM) degradation. The combined analysis of both ChIP-Seq and RNA-Seq results showed that KLF14 might play very important roles in the regulation of proliferation and cancer, lipid metabolic process, and immune system process and immune response.

KLF14 has been verified as an important regulator in the lipid metabolism [18, 23]. GWAS has suggested that a single nucleotide polymorphism (SNP) in one of the KLF14 locus is associated with low HDL-C and TGs in European populations [20]. The possible mechanism underlying the function of KLF14's regulating effect of lipid metabolism may be related with transcriptional activation of the SK1 promoter, which can lead to the up-regulation of sphingosine-1-phosphate (S1P) lipid signaling [18]. In addition, KLF14 regulates plasma HDL-C levels and cholesterol efflux capacity by mediating hepatic apoA-I production [24]. Our study results showed that cholesterol, HDL-C, LDL-C, and TGs were significantly decreased in KLF14-/- mice, which was partially consistent with previous study results. The reasons for this difference might be associated with the different functions of KLF4 between mice and humans; it might also be due to the housing environment and timing differences between mice and humans.

In addition to the important role in lipometabolism pathways, KLF14 may significant ly influence the immune functions. The latest development has shown that KLF14 is of high expression in the lymphatic organs, which revealed that KLF14 may play an important part in driving $T$ cell differentiation s. The underlying mechanism may be that KLF14 can bind to the Treg-specific demethylation region (TSDR) enhancer locus of FOXP3, thereby epressing the expression of the immune gene FOXP3 [25]. The knockdown of KLF14 has been shown to greatly decrease the levels of some inflammatory cytokines, such as IL-6, TNF- $a$ and MCP-1 in apoe-/- mice fed a high-fat diet. However, KLF14 over-expression clearly suppressed the secretion of those cytokines in RAW264.7 cells. The effect of KLF14 on Ac-LDLinduced inflammatory cytokine secretion in macrophages at least partly concerns the p38 MAPK and extracellular signal-regulated

Page 5/13 
kinase 1 (ERK1)/2 signaling pathways [26]. Our study also revealed that some inflammatory genes, such as Ccl28, Socs2, Jchain, and $\mathrm{Cfd}$, were directly or indirectly regulated by KLF14. The level of Socs2 is closely related to KLF14, but the dual-luciferase reporter assay of Socs2 has failed to find that KLF14 can directly regulate the expression of Socs2. The mechanism of KLF14 regulation of Socs 2 needs to be further clarified.

The function of KLF14 in proliferation and tumorigenesis has also been reported. Previous studies have demonstrated that KLF14induced expression of alk-1may be involved in the TGF- $\beta 1$-mediated cell proliferation in Leydig cells [27]. A recent study revealed that KLF14 knockout mice showed an induction pf centrosome amplification, chromosome aneuploidy, and spontaneous tumorigenesis, which was mainly caused by transcriptional inhibition of Plk4, while Plk4 overexpression could induce centrosome overduplication [13]. Our study showed that glomerular proliferation was significantly aggravated in the kidneys of KLF14-/- compared with wild-type mice. Similar to the histological manifestations, the number of glomerular nucleated cells increased more noticeably in the KLF14-/- mice. Furthermore, cell experiments also confirmed that KLF14 overexpression in renal mesangial cells could significantly reduce the rate of EdU positivity, suggesting that KLF4 had an anti-proliferative function in the kidneys.

To further confirm the target genes of KLF14, ChIP-Seq was used to screen its target genes. The ChIP-Seq and RNA-Seq results in KLF14-/- mice were combined for analysis. The results revealed five overlapping target genes between the RNA-Seq and Chip-Seq results. These target genes were Socs2, Btg2, Hdc, Ler2, and Akr1b3, among which, Btg2 was associated with the regulation of proliferation. We further performed bioinformatics analyses, the dual-luciferase reporter assay, and the EMSA assay to confirm that Btg2 was a target gene of KLF14, which is a member of the BTG/TOB family. Recent studies have indicated that all TOB/BTG family members are tumor suppressor genes and that this family is closely related to other tumor suppressor genes such as p53 [28]. Btg2 is located on band 3, region 2 of the long arm of chromosome 1 and is a protein consisting of 158 amino acids. Btg2 is an immediate early response gene. When cells are stimulated by genotoxic drugs, oxidative stress, and ionizing radiation, Btg2 expression is up-regulated through p53dependent or independent pathways to act on a series of downstream target molecules, causing cells to arrest at G1/S phase, which is the major functional pathway of the Btg2 gene. A large number of studies have confirmed that Btg2 expression is down-regulated in many tumors. As a tumor suppressor gene, Btg2 is widely inloved in differentiation, proliferation, DNA damage repair, and apoptosis of various tumor cells [29-31].

To clarify whether KLF14 inhibited the proliferation of mouse renal mesangial cells by regulating the target gene Btg2, a Btg2 gene siRNA was synthesized and transfected into the cells. The results showed that the anti-proliferative effect of KLF14 on mesangial cells was significantly diminished after transfection of Btg2 siRNA. This result indicated that Btg2 activation was dispensable for KLF14-induced anti-proliferation.

In short, the transcriptome sequencing and ChIP-Seq results in this study confirmed that KLF14 mainly regulated metabolism, proliferation, and inflammation-related processes. Expression of the Btg2 gene in mouse kidneys was regulated by KLF14, and Btg2 gene expression decreased after KLF14 knockout. The combined results of the luciferase reporter gene assay and EMSA herein confirmed that Btg2 was a target gene of KLF14. In vitro studies showed that KLF14 could inhibit renal mesangial cell proliferation by regulating the activity of Btg2. Thus, we can get the conclusion that KLF14 might exert its function in kidney tissues by regulating metabolism, proliferation, and inflammation signaling pathways in cells. KLF14 inhibited the proliferation of primary renal mesangial cells by promoting expression of its target gene Btg2. This study provides a basis for further studies of the mechanism of KLF14 in the development of kidney diseases, especially in mesangial proliferative glomerulonephritis, metabolic nephropathy, and kidney tumors.

\section{Materials And Methods}

\section{Construction of KLF14-deficient mice models}

KLF14-deficient mice were generated by Cyagen Biosciences Inc. (Guangzhou, China) using the genome editing technology TALEN. A pair of TALEN constructs for KLF14 knockout were cloned into a mammalian expression vector, pCMV-TALEN (Origene, USA), and capped, polyA-tailed messenger RNA (mRNA) for injection was produced using the Ambion mMessage mMachine kit (Thermo Fisher Scientific, USA). The knockout (KO) mice were produced by microinjecting TALEN mRNAs into fertilized eggs from C57BL/ 6 strains. The $\mathrm{KO}$ alleles were sequenced and validated. Wild type C57BL/6 (KLF14+/+) mice were purchased from Vital River Laboratories (Beijing, China). All animals were housed in cages at constant room temperature $\left(20^{\circ} \mathrm{C}\right)$ and humidity $(70 \%)$ under a controlled light-dark cycle in the Experimental Animal Center of Chinese PLA General Hospital. The experimental protocol was carried out in accordance with X Lian et al. 712 British Journal of Pharmacology (2019) 176 711-724 the approved guidelines of the Institutional Animal Care and Use Committee at the Chinese PLA General Hospital. Animal studies are reported in compliance with the ARRIVE guidelines (Kilkenny et al.,

Page 6/13 
2010). Kilkenny C, Browne W, Cuthill IC, Emerson M, Altman DG (2010).Animal research: reporting in vivo experiments: the ARRIVE guidelines. Br J Pharmacol 160: 1577-1579.

\section{Analysis of blood and urine biochemical parameters in KLF14-deficient mice}

At the end of the study, when these mice were one year old, blood samples were collected in microcentrifuge tubes from animals in the KLF14-deficient mice group and wild-type group. Plasma was separated immediately and stored at $-80^{\circ} \mathrm{C}$ until used for further measurements. The biochemical analyzer (Erba Chem 7, Germany) was used along with specific diagnostic kits (Transasia Biomedicals Ltd, India) for detection of plasma glucose, albumin, creatinine, blood urea, cholesterol, HDL-C, low-density lipoprotein cholesterol (LDLC) and triglycerides (TGs). Random urine samples of mice were tested for the ratio between urine protein and urine creatinine. The concentration of albumin in urine was quantified by ELISA (Exocell, Philadelphia, PA), and urine creatinine was determined using the creatinine assay kit (Diazyme Laboratories, Poway, CA).

\section{Kidney histological pathological analysis in KLF14-deficient mice}

At the time of euthanasia, kidneys from the two groups were used for pathologic examination. One kidney,which was freshly frozen was ued for gene expression analysis, and the other was fixed in $10 \%$ neutral buffered formalin. The formalin-fixed tissue was embedded in paraffin, and 4-mm sections were stained by periodic acid-Schiff (PAS) staining. The numbers of cells in at least 20 glomeruli per murine kidney section were counted to quantify the degree of cell proliferation [14].

\section{Immunohistochemical analysis in KLF14-deficient mice}

Proliferating cell nuclear antigen (PCNA) immunohistochemical staining,which is based on the previous literature[15],was used to detect the degree of cell proliferation. The presence of PCNA protein was immunohistochemically demonstrated in tissue specimens by the indirect avidin-biotinylated peroxidase complex method (ABC Kit, Burlingame, CA, USA) with rabbit anti-PCNA antibody (Santa Cruz Biotechnology, USA). The primary antibody was replaced with PBS for negative controls,.

\section{Analysis of gene expression profiles in the kidneys of KLF14-deficient mice by RNA-Seq}

Three mice from each animal in the KLF14-deficient mice group and wild-type group were collected to extract RNA from kidney tissues, which was sent to BGI (Beijing Genomics Institute, Shenzhen, Guangdong, China) for RNA-SEq. Complementary DNA (cDNA) libraries were prepared using the Illumina TruSeq RNA Library Preparation kit (Illumina, San Diego, CA). The quality of the cDNA library was assessed with the Agilent 2100 Bioanalyzer (Agilent Technologies Co. Ltd., Santa Clara, CA, USA). In the end, the six libraries were sequenced with an Illumina Hiseq 2000.

Gene expression levels were quantified using the RSEM software package [15]. The NOISeq method was adopted to screen the differentially expressed genes (DEGs) between KLF14-deficient and KLF14 wild-type samples. Annotation analysis of Gene Ontology (GO) was performed for the screened DEGs. After GO annotation was obtained for DEGs, WEGO software [16] was used to conduct GO functional classification. Pathway enrichment analysis of DEGs was performed based on the Kyoto Encyclopedia of Genes and Genomes (KEGG) database [17]. Three biological replicates were assessed in this study. Statistical analysis was performed, and DEGs were selected based on a fold-change $\geq 2$ and q-value $(\%)<5 \%$.

\section{Validation of mRNA expression levels using quantitative real-time polymerase chain reaction (qRT-PCR)}

According to the TRIzol protocol (Invitrogen Life Technologies, Carlsbad, CA), total RNA was isolated from the renal cortex. Then cDNA was synthesized with reverse transcription reagents (Takara, Japan). iQSYBR Green Supermix (Bio-Rad Laboratories, Hercules, CA) was used for real-time PCR (ABI Prism TM 7500 Sequence Detection System; Perkin-Elmer Applied Biosystems). The standard curve method was used to calculated the levels of mRNA expression. The $\mathrm{Ct}$ values were used to assessted the levels of mRNA expression. Analytical data were adjusted based on the $\beta$-actin mRNA expression levels as an internal control. The results were analyzed using the $2-\triangle \Delta C T$ method. The primers used are described in Table S3.

\section{Screening of KLF14 target genes using ChIP-Seq}

To identify target genes of KLF14, ChIP assay was performed using the EZ-Magna-ChIP kit (Millipore, USA) with the kidney tissues of wild-type KLF14 mouse. The resulting nuclear extract was sonicated on wet ice and then immunoprecipitated with the appropriate KLF14 antibody (sc-292659, Santa Cruz Biotechnology, USA). The specificities of ChIP of the antibody were tested by PCR amplification of the 
promoter of the sphingosine kinase 1 (SK1) gene, which has been validated to be directly regulated by KLF14 [18]. The upstream primer sequence was 5'-CAC CTC CTA AGA CCA CTC TC-3', and the downstream primer sequence was 5'-ACT GGC ATC TTT CTT GAT CG-3'.

For ChIP-Seq, $10 \mathrm{ng}$ immunoprecipitated DNA fragments were used to prepare libraries with the NEBNext RNA Library Prep Kit (New England Biolabs, USA) and the Ovation SP Ultralow DR Multiplex System (NuGEN, USA) following the manufacturer's protocol. Libraries were sequenced for 50 cycles on a HiSeq 2000 or HiSeq2500 Illumina instrument. The GO database is currently an important tool for gene function analysis. The method used in this study was mainly based on the GO Term Finder

(http://www.yeastgenome.org/help/analyze/GO-term-finder). Peak related genes were compared to the G0 term database

(http://www.geneontology.org/), and the number of genes of each term was calculated. Next, the hypergeometric test was applied to identify the GO term that had significant enrichment of peak related genes compared with the whole genome background. KEGG is a major public database for pathway analysis. In this study, pathway significant enrichment analysis was based on the KEGG pathway and revealed pathways with significant enrichment of peak related genes compared with the whole genome background. The major calculation method was the same as that applied for GO analysis.

\section{Validation of KLF14 target genes using the dual-luciferase reporter gene assay}

\section{Construction of the luciferase reporter vector}

To validate that Btg2 was in fact a target gene of KLF14, the promoter sequence (2000 bp upstream of $5^{\prime} U T R$ ) of the Btg2 gene was first amplified using PCR. The upstream primer sequence for PCR amplification was 5'-GCG TGC TAG CCC GGG CTC GAG GTC GTT TGT AGA AAA TGA TGA AAT GTC TTG CTG GT-3', and the downstream primer sequence was 5'-CAG TAC CGG AAT GCC AAG CTT GGG GAG GCG GGG AGC CGT CGG TGG CGC-3'. The restriction endonuclease sites Xhol and HindIII and protective bases were added at the termini of the upstream and downstream primers. The PCR product was cloned into the multiple cloning sites upstream of the luciferase gene in the luciferase reporter gene vector PGL3-Basic (Promega, USA). This plasmid was named the pGL3-Basic-Btg2 promoter. The positive clones were sequenced, analyzed, and compared.

The sequencing revealed that target gene expression plasmid was successfully constructed. The constructed plasmid was subjected to ultra-pure endotoxin-free extraction.

\section{Cell transfection and dual-luciferase reporter assay}

Human embryonic kidney (HEK) 293 cells were purchased from the American Type Culture Collection (ATCC). All cell culture products were purchased from Invitrogen Gibco. HEK293 cells were cultured in a $5 \% \mathrm{CO}_{2}$ humidified incubator at $37^{\circ} \mathrm{C}$. Cells $(\sim 60 \%$ confluence $)$ were transfected using jet PRIMETM (Polyplus-transfection, USA) for $48 \mathrm{~h}$. The mouse KLF14 eukaryotic expression vector pCMV6-ACGFP-KLF14 (MG221172) and the KLF14 empty vector pCMV6-AC-GFP (PS100010) were purchased from Origene (Origene, USA). For the reporter assays, the cells were cultured in a 24-well plate and then co-transfected with the dual luciferase reporter plasmid pGL3-BasicBtg2 promoter or pGL3-Basic, pCMV6-AC-GFP-KLF14 or pCMV6-AC-GFP and the renilla luciferase reporter gene plasmid (pGMLR-TK luciferase reporter plasmid). Firefly and renilla luciferase activities were measured using the Dual-Luciferase Reporter Assay System (E1910, Promega, USA). Renilla luciferase activity was normalized to firefly luciferase activity. Each experiment was repeated at least three times.

\section{Nuclear extraction and EMSA}

Nuclear protein was extracted from the renal cortex, and EMSA was performed with the LightShift Chemiluminescent EMSA kit (Thermo Scientific, USA) according to the instructions. In Brief, $15 \mu \mathrm{g}$ nuclear extract was incubated with biotin labeled-probe or unlabeled probe in a final volume of $20 \mu \mathrm{l}$ binding reaction containing $1 \mu \mathrm{g}$ poly ( $\mathrm{dl}-\mathrm{dC}$ ) and the binding buffer. The 5 '-biotin end-labeled oligonucleotides that corresponded to the KLF14 transcription factor recognition sequences within the Btg2 promoter region were synthesized by GenePharma Co., Ltd. (Shanghai, China) and used as probes. The probe sequences were as follows: KLF14-labeled probe; GGG CAC GCC CCC AT; KLF14 cold probe: GGG CAC GCC CCC AT. KLF14 mut probe: GGG TTA GAT CAC AT. Samples were subjected to $6 \%$ native polyacrylamide gel in a $0.5 \times$ Tris borate-EDTA buffer at $100 \mathrm{~V}$ for $3 \mathrm{~h}$ and then transferred to a nylon membrane in $0.5 \times$ Tris borate-EDTA buffer at $100 \mathrm{~V}$ for $1 \mathrm{~h}$. After UV crosslinking, probing with streptavidin-conjugated horseradish peroxidase (S-HRP), and incubating with substrates, the nylon membrane was exposed to X-ray film. Competition EMSA was then performed. 


\section{Cell culture and cell proliferation assay}

Primary mouse renal mesangial cells (MRMCs) were purchased from ScienCell Research Laboratories (\#M4200-57, ScienCell, USA) and were maintained in Mesangial Cell Medium (MCM, \#4201, ScienCell,USA), which was supplemented with $10 \mathrm{ml}$ of fetal bovine serum (FBS No. 0010), $5 \mathrm{ml}$ of mesangial cell growth supplement (MsCGS, No. 4252), and $5 \mathrm{ml}$ of penicillin/streptomycin solution (P/S, No.0503) in a humidified $5 \% \mathrm{CO}_{2}$ atmosphere. Cells were synchronized by incubation with $0.1 \%$ FBS-MCM for $24 \mathrm{~h}$.

To determine the effect of KLF14 on glomerular cell proliferation, the mouse KLF14 eukaryotic expression vector pCMV6-AC-GFP-KLF14 (MG221172, Origene, USA) and KLF14-expressing control empty vector pCMV6-AC-GFP (PS100010, Origene, USA) were transfected into primary MRMCs. At $48 \mathrm{~h}$ after transfection, these cells were collected, and the cell proliferation assay was performed. Cell proliferation was analyzed with the Click-iT® Plus EdU Alexa Fluor ${ }^{\circledR} 555$ Imaging Kit (C10638, Invitrogen, USA) according to the previous literature[15].

\section{Western blot analysis}

The method was based on previous literature[15].In brief the glomeruli and cells were lysed in RIPA buffer containing a protease inhibitor cocktail. Protein concentrations were determined using a BCA Protein Assay Kit (Thermo Fisher Scientific, USA). In total, $30 \mu \mathrm{g}$ of total protein per sample was transferred to membranes, which were incubated overnight at $4{ }^{\circ} \mathrm{C}$ with primary antibodies against Btg2,cyclinD1, cyclin B1, CDK2, CDK4, cyclin E1 (Abcam, USA) and B-actin (Abcam, USA). The samples were then incubated with secondary antibody. Image $\mathrm{J}$ software was used for blot analysis. The intensities of the protein bands are presented as ratios to that of $\beta$-actin, and data from the control group were arbitrarily set as 1.0.

\section{Statistical analysis}

Statistical analysis was performed using IBM SPSS Statistics 17.0.2 software (IBM Corporation, Armonk, NY, USA). The results are presented as the mean values \pm standard deviation (SD). The Student's t-test was used to compare differences between the means of two groups. Multiple comparisons of parametric data were performed using one-way analysis of variance (ANOVA). A p-value $<0.05$ was considered significant.

\section{Declarations}

\section{Acknowledgements}

This work was supported by a grant (No. 81570659 81770663 ) from the National Natural Science Foundation of China, a grant (No. 2016YFA0101002) from the National Key Research and Development Program of China and a grant (No. 20158332) from the Natural Science Foundation of Hainan Province.

\section{Disclosure Statement}

No conflicts of interest.

\section{Author Contribution}

Xue-Yuan Bai designed the experiments. Xiaoyuan Wu, Yuxiang Ma and Xiaoying Lian performed the experiments. Shupeng Lin and Shunlai Shang analysed the data. Xiaoyuan Wu wrote the manuscript. Xue-Yuan Bai critically revised the manuscript.

\section{Data Availability Statement}

The data that support the findings of this study are available from the corresponding author upon reasonable request.

\section{References}

1. Zhao, C. \& Meng, A. Sp1-like transcription factors are regulators of embryonic development in vertebrates. Dev Growth Differ. 47 (4), 201-211 (2005). 
2. Fernandez-Zapico, M. E. et al. A functional family-wide screening of SP/KLF proteins identifies a subset of suppressors of KRASmediated cell growth. Biochem J. 435 (2), 529-537 (2011).

3. Brey, C. W., Nelder, M. P., Hailemariam, T., Gaugler, R. \& Hashmi, S. Kruppel-like family of transcription factors: an emerging new frontier in fat biology. Int J Biol Sci. 5 (6), 622-636 (2009).

4. Ghaleb, A. M. et al. Kruppel-like factors 4 and 5: the yin and yang regulators of cellular proliferation. Cell Res. 15 (2), $92-96$ (2005).

5. Suske, G., Bruford, E. \& Philipsen, S. Mammalian SP/KLF transcription factors: bring in the family. Genomics. 85 (5), $551-556$ (2005).

6. Black, A. R., Black, J. D. \& Azizkhan-Clifford, J. Sp1 and kruppel-like factor family of transcription factors in cell growth regulation and cancer. J Cell Physiol. 188 (2), 143-160 (2001).

7. McConnell, B. B. \& Yang, V. W. Mammalian Kruppel-like factors in health and diseases. Physiol Rev. 90 (4), 1337-1381 (2010).

8. Bieker, J. J. Kruppel-like factors: three fingers in many pies. J Biol Chem. 276 (37), 34355-34358 (2001).

9. Parker-Katiraee, L. et al. Identification of the imprinted KLF14 transcription factor undergoing human-specific accelerated evolution. PLoS Genet. 3 (5), e65 (2007).

10. Teslovich, T. M. et al. Biological, clinical and population relevance of 95 loci for blood lipids. Nature. 466 (7307), 707-713 (2010).

11. Banno, M. et al. A case control association study and cognitive function analysis of neuropilin and tolloid-like $1 \mathrm{gene}$ and schizophrenia in the Japanese population. PLoS One. 6 (12), e28929 (2011).

12. Sladek, R. et al. A genome-wide association study identifies novel risk loci for type 2 diabetes. Nature. 445 (7130), $881-885$ (2007).

13. Fan, G. et al. Loss of KLF14 triggers centrosome amplification and tumorigenesis. Nat Commun. 6, 8450 (2015).

14. Abrera-Abeleda, M. A., Xu, Y., Pickering, M. C., Smith, R. J. \& Sethi, S. Mesangial immune complex glomerulonephritis due to complement factor D deficiency. Kidney Int. 71 (11), 1142-1147 (2007).

15. Li, B. \& Dewey, C. N. RSEM: accurate transcript quantification from RNA-Seq data with or without a reference genome. BMC Bioinformatics. 12, 323 (2011).

16. Jie Gao. Knockdown of Cxcl10 Inhibits Mesangial Cell Proliferation in Murine Habu Nephritis Via ERK Signaling Cell. Physiol Biochem. 42, 2118-2129 (2017).

17. Ye, J. et al. WEGO: a web tool for plotting GO annotations.Nucleic Acids Res. 2006;34(Web Server issue) :W293-7.

18. Kanehisa, M. et al. KEGG for linking genomes to life and the environment. Nucleic Acids Res. 36 (Database issue), D480-4 (2008).

19. de Assuncao, T. M. et al. New role for Kruppel-like factor 14 as a transcriptional activator involved in the generation of signaling lipids. J Biol Chem. 289 (22), 15798-15809 (2014).

20. Teslovich, T. M. et al. Biological, clinical and population relevance of 95 loci for blood lipids. Nature. 466 (7307), 707-713 (2010).

21. Anunciado-Koza, R. P., Manuel, J. \& Koza, R. A. Molecular correlates of fat mass expansion in C57BL/6J mice after short-term exposure to dietary fat. Ann N Y Acad Sci. 1363, 50-58 (2016).

22. Koza, R. A. et al. Changes in gene expression foreshadow diet-induced obesity in genetically identical mice. PLoS Genet. 2006;2(5):e81.b

23. Yang, M. et al. Kruppel-like factor 14 increases insulin sensitivity through activation of PI3K/Akt signal pathway. Cell Signal. 27 (11), 2201-2208 (2015).

24. Civelek, M. \& Lusis, A. J. Conducting the metabolic syndrome orchestra. Nat Genet. 43 (6), 506-508 (2011).

25. Guo, Y. et al. Perhexiline activates KLF14 and reduces atherosclerosis by modulating ApoA-I production. J Clin Invest. 125 (10), 3819-3830 (2015).

26. Sarmento, O. F. et al. A novel role for KLF14 in T regulatory cell differentiation. Cell Mol Gastroenterol Hepatol. 1 (2), $188-202$ e4. (2015).

27. Wei, X. et al. A novel role for the Kruppel-like factor 14 on macrophage inflammatory response and atherosclerosis development. Cardiovasc Pathol. 27, 1-8 (2017).

28. Gonzalez, C. R., Vallcaneras, S. S., Calandra, R. S. \& Gonzalez Calvar, S. I. Involvement of KLF14 and egr-1 in the TGF-beta1 action on Leydig cell proliferation. Cytokine. 61 (2), 670-675 (2013).

29. Mao, B., Zhang, Z. \& Wang, G. BTG2: a rising star of tumor suppressors (review). Int J Oncol. 46 (2), $459-464$ (2015).

30. Gao, S. S., Yang, X. H. \& Wang, M. Inhibitory effects of Bcell translocation gene 2 on skin cancer cells via the Wnt/betacatenin signaling pathway. Mol Med Rep. 14 (4), 3464-3468 (2016).

Page 10/13 
31. Choi, O. R., Ryu, M. S. \& Lim, I. K. Shifting p53-induced senescence to cell death by TIS21(/BTG2/Pc3) gene through posttranslational modification of p53 protein. Cell Signal. 28 (9), 1172-1185 (2016).

32. Lim, S. K., Choi, Y. W., Lim, I. K. \& Park, T. J. BTG2 suppresses cancer cell migration through inhibition of Src-FAK signaling by downregulation of reactive oxygen species generation in mitochondria. Clin Exp Metastasis. 29 (8), 901-913 (2012).

\section{Figures}

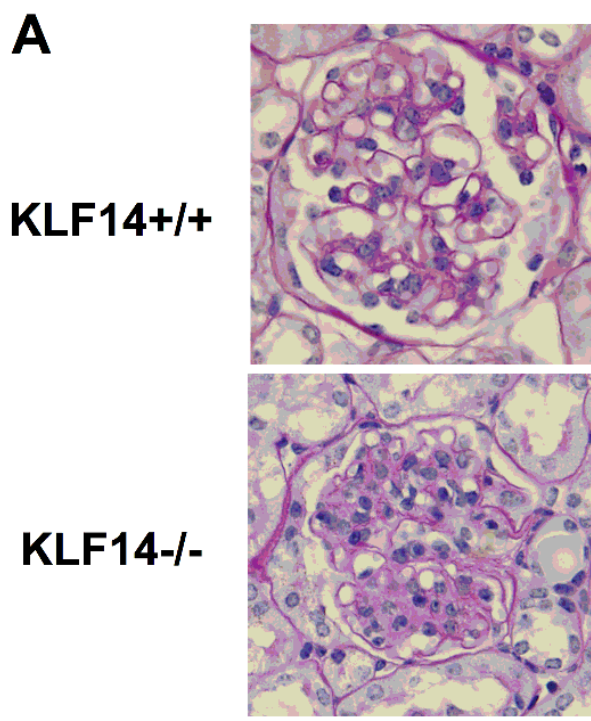

B
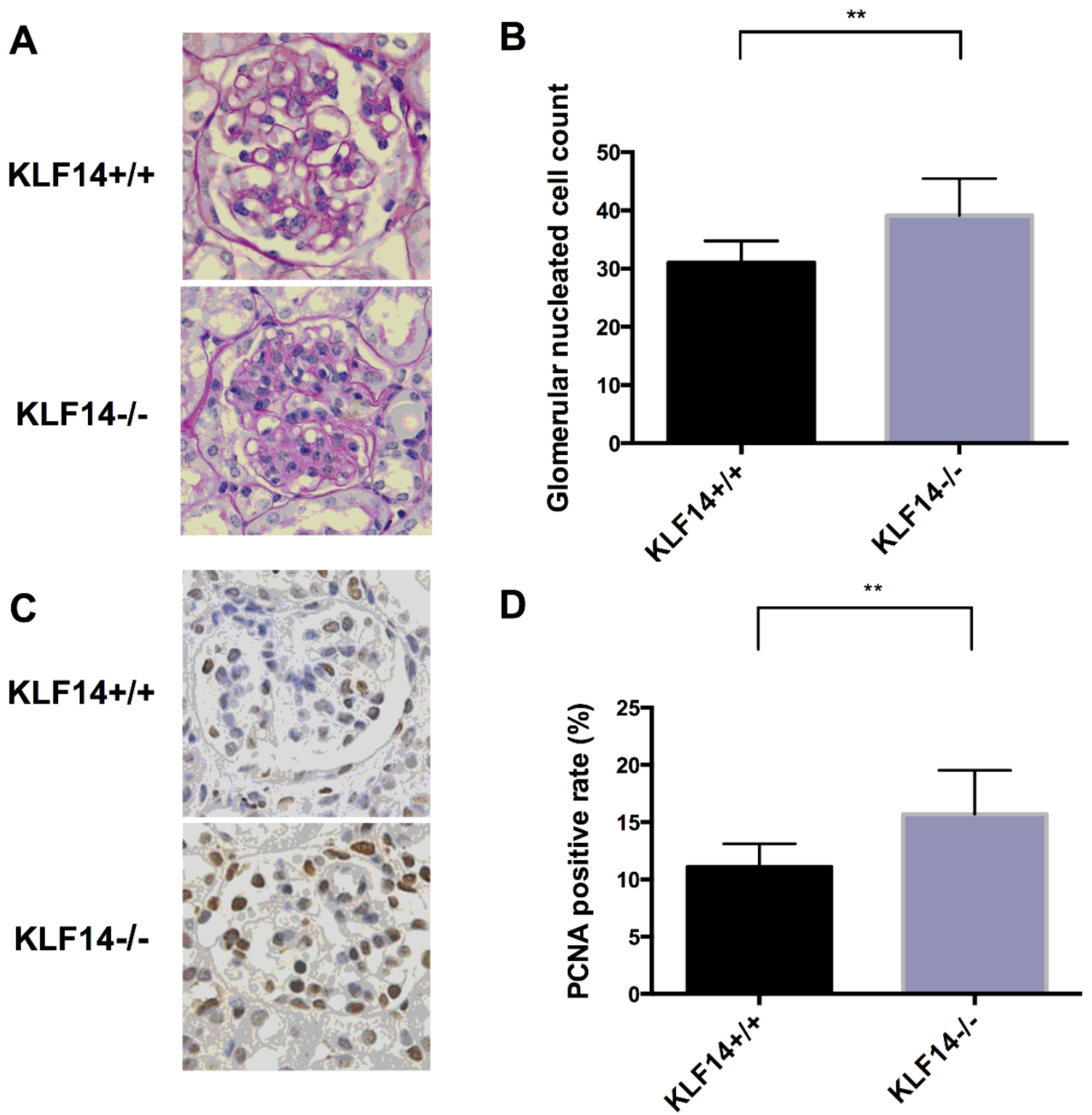

\section{Figure 1}

Pathological changes in kidney tissues of KLF14-/- mice. (A) PAS staining of renal tissues of KLF14+/+ mice and KLF14-/- mice. KLF14-/- mice exhibited more extracellular matrix accumulation and cell proliferation. (B) Glomerular nucleated cells in both KLF14+/+ and KLF14- $/$ - mice were counted ( $\mathrm{n}=8$ per group and 20 glomeruli per mouse). The results are expressed as the mean values \pm SD; ${ }^{*} \mathrm{p}<0.01$ versus KLF14+/+ group. (C) PCNA immunohistochemistry of glomeruli in both KLF14+/+ and KLF14-/- mice to evaluate the cell proliferation rate. (D) The PCNA-positive rate was higher in KLF14-/- than in KLF14+/+mice ( $n=7$ per group and 20 glomeruli per mouse). The results are expressed as the mean values $\pm \mathrm{SD} ;{ }^{\star *} \mathrm{p}<0.01$ versus $\mathrm{KLF} 14+/+$ group. 

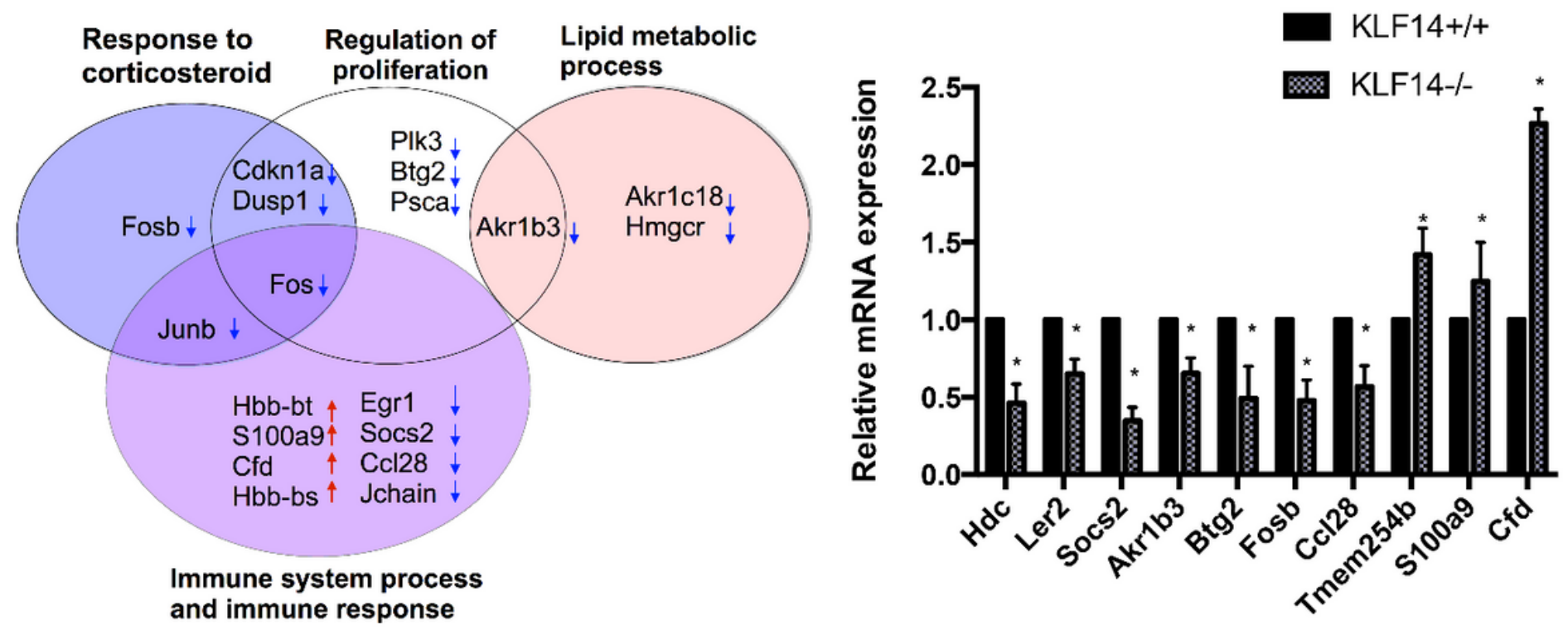

Figure 2

Changes in gene expression profile in the kidneys of KLF14 -/- mice. (A)The major functional categories of DEGs in kidney tissues between KLF14-/- and KLF14+/+ mice. Red arrows pointing up indicate DEGs that were up-regulated in the kidney of KLF14-/- mice; blue arrows pointing down represent DEGs that were down-regulated in the kidney of KLF14-/- mice. (B) Validation of mRNA expression levels using PCR. The results showed that the mRNA expression levels of Btg2, Akr1b3, Hdc, ler2, Socs2, Fosb, and Ccl28 in the KLF14-/- group were significantly increased compared with the KLF14+/+ group; the mRNA expression levels of Tmem254b, S100a9, and Cfd were significantly decreased. $p<0.05$ versus KLF14+/+ group.

Figure 3

Combined analyses of screening results of KLF14 target genes using RNA-Seq and ChIP-Seq. (A) The numbers of differential genes detected by ChIP-Seq and RNA-Seq. The blue circles represent the peak related genes of KLF14 identified by ChIP-Seq, white circles represent the genes identified by RNA-Seq, and the intersections between blue and white circles contain their shared genes. (B)Gene pathway network analysis for Socs2, Btg2, Akr1b3, Hdc, and ler2.

Figure 4

Confirmation of Btg2 as a target gene of transcription factor KLF14. (A) Either dual-luciferase reporter plasmid (pGL3-Basic-Btg2 promoter or pGL3-Basic) and pCMV6-AC-GFP-KLF14 plasmid or pCMV6-AC-GFP were transfected into HEK293 cells. Renilla luciferase activity was normalized to firefly luciferase activity. The results shown are the mean \pm SEM of triplicate determinations from one of three identical experiments. ${ }^{*} \mathrm{p}<0.05$ versus Btg2+KLF14 control group. (B) EMSA of KLF14 to analyze the transcription factor binding site in the Btg2 promoter. The 5-biotin end-labeled probe was incubated in the absence (lane 1) or presence (lane 3) of nuclear extracts from primary mouse renal mesangial cells. Unlabeled cold probe (lane 4) and cold mutated probe (lane 5) were used as competitors. The 5biotin end-labeled probe was incubated in the presence of nuclear extracts from primary MRMCs that were transfected with KLF14 (lane 2). 


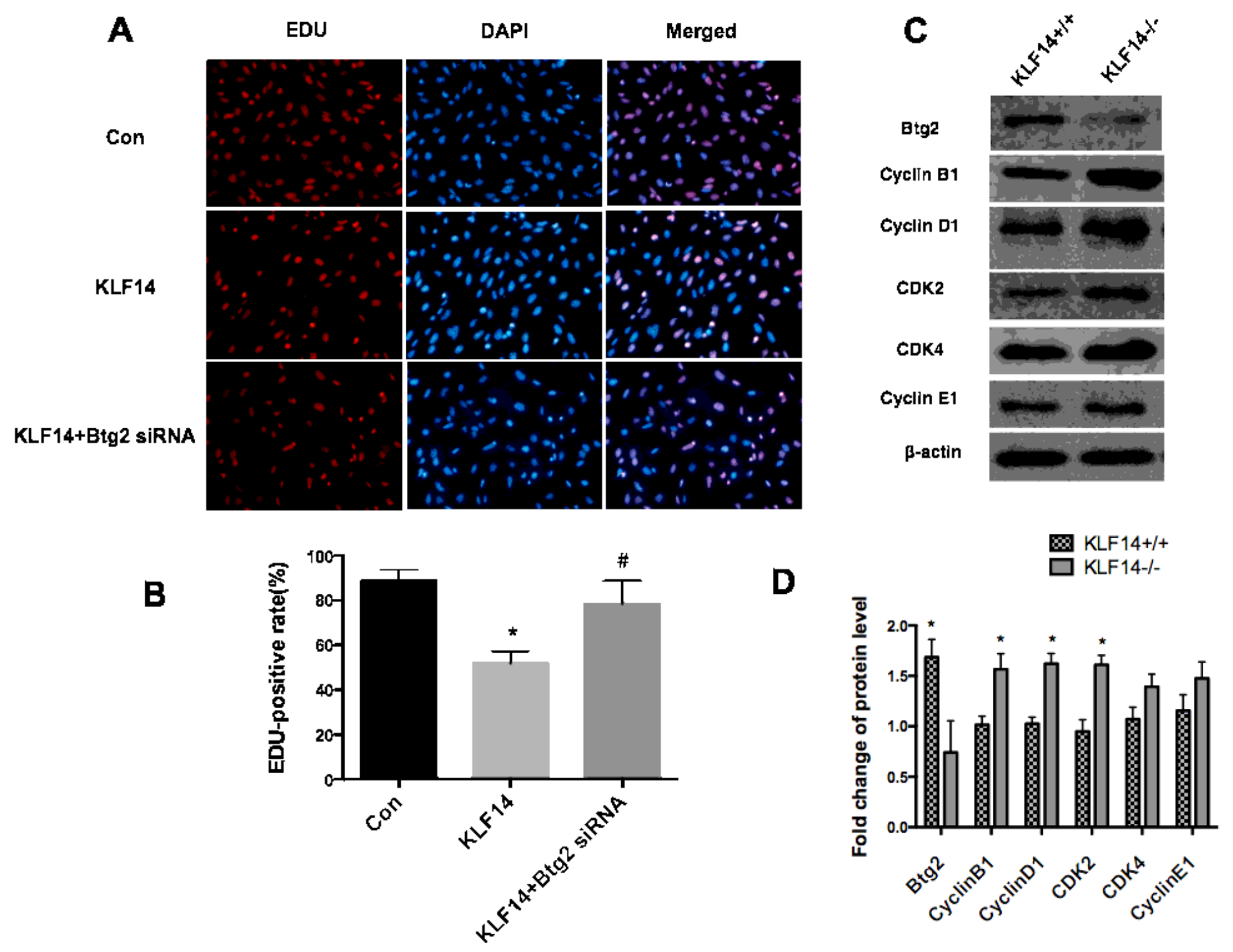

Figure 5

Effects of KLF14 up-regulation and down-regulation on proliferation. (A) EdU incorporation assay of MRMC proliferation to assess effects of KLF14 up-regulation and Btg2 inhibition on MRMC proliferation. (red, EdU; blue, DAPI; pink, merge). One representative experiment is shown. (B) The percentage of EdU-positive cells among the total number of cells represents the degree of proliferation. The results are expressed as the mean values $( \pm S D) ;{ }^{*} p<0.05$ versus the control group, $\# p<0.05$ versus the KLF14 group. (C) Western blot analysis of Btg2, cyclin B1, cyclin D1, cyclin E1,CDK2 and CDK4 protein expression of kidneys in both KLF14+/+ and KLF14-/- mice. (D) The expression levels of positive cell cycle regulatory proteins were quantified by densitometry. The intensities of the protein bands were presented as ratios to those of the $\beta$-actin bands; Results are expressed as mean values ( $\pm S D)$; ${ }^{*} P<0.05$ versus the KLF14+/+group.

\section{Supplementary Files}

This is a list of supplementary files associated with this preprint. Click to download.

- SupplementaryInformation.docx 